\title{
Strong convergence theorems by a hybrid extragradient-like approximation method for asymptotically nonexpansive mappings in the intermediate sense in Hilbert spaces
}

Eskandar Naraghirad ${ }^{1}$, Ngai-Ching Wong ${ }^{2^{*}}$ and Jen-Chih Yao $^{3}$

* Correspondence: wong@math. nsysu.edu.tw

${ }^{2}$ Department of Applied Mathematics, National Sun Yat-Sen University, Kaohsiung 804, Taiwan Full list of author information is available at the end of the article

\begin{abstract}
Let $\mathrm{C}$ be a nonempty closed convex subset of a real Hilbert space $H$. Let $S: C \rightarrow C$ be an asymptotically nonexpansive map in the intermediate sense with the fixed point set $F(S)$. Let $A: C \rightarrow H$ be a Lipschitz continuous map, and $V I(C, A)$ be the set of solutions $u \in C$ of the variational inequality

$$
\langle A u, v-u\rangle \geq 0, \quad \forall v \in C .
$$

The purpose of this study is to introduce a hybrid extragradient-like approximation method for finding a common element in $F(S)$ and $V I(C, A)$. We establish some strong convergence theorems for sequences produced by our iterative method.

AMS subject classifications: 49J25; 47H05; 47H09.

Keywords: asymptotically nonexpansive mapping in the intermediate sense, variational inequality, hybrid extragradient-like approximation method, monotone mapping, fixed point, strong convergence
\end{abstract}

\section{Introduction}

Let $H$ be a real Hilbert space with inner product $(\cdot, \cdot)$ and norm $\|\cdot\|$, respectively. Let $C$ be a nonempty closed convex subset of $H$ and let $P_{C}$ be the metric projection from $H$ onto $C$. A mapping $A: C \rightarrow H$ is called monotone [1-3] if

$$
\langle A u-A v, u-v\rangle \geq 0, \quad \forall u, v \in C ;
$$

and $A$ is called $k$-Lipschitz continuous if there exists a positive constant $k$ such that

$$
\|A u-A v\| \leq k\|u-v\|, \quad \forall u, v \in C .
$$

Let $S$ be a mapping of $C$ into itself. Denote by $F(S)$ the set of fixed points of $S$; that is $F(S)=\{u \in C: S u=u\}$. Recall that $S$ is nonexpansive if

$$
\|S u-S v\| \leq\|u-v\|, \quad \forall u, v \in C ;
$$

and $S$ is asymptotically nonexpansive [4] if there exists a null sequence $\left\{\gamma_{n}\right\}$ in $[0,+$ $\infty)$ such that 


$$
\left\|S^{n} u-S^{n} v\right\| \leq\left(1+\gamma_{n}\right)\|u-v\|, \quad \forall u, v \in C \text { and } n \geq 1 .
$$

We call $S$ an asymptotically nonexpansive mapping in the intermediate sense [5] if there exists two null sequences $\left\{\gamma_{n}\right\}$ and $\left\{c_{n}\right\}$ in $[0,+\infty)$ such that

$$
\left\|S^{n} x-S^{n} y\right\|^{2} \leq\left(1+\gamma_{n}\right)\|x-y\|^{2}+c_{n}, \quad \forall x, y \in C, \forall n \geq 1 .
$$

Let $A: C \rightarrow H$ be a monotone and $k$-Lipschitz continuous mapping. The variational inequality problem [6] is to find the elements $u \in C$ such that

$$
\langle A u, v-u\rangle \geq 0, \quad \forall v \in C .
$$

The set of solutions of the variational inequality problem is denoted by $\operatorname{VI}(C, A)$. The idea of an extragradient iterative process was first introduced by Korpelevich in [7]. When $S: C \rightarrow C$ is a uniformly continuous asymptotically nonexpansive mapping in the intermediate sense, a hybrid extragradient-like approximation method was proposed by Ceng et al. [8, Theorem 1.1] to ensure the weak convergence of some algorithms for finding a member of $F(S) \cap V I(C, A)$. Meanwhile, assuming $S$ is nonexpansive, Ceng et al. in [9] introduced an iterative process and proved its strong convergence to a member of $F(S) \cap V I(C, A)$.

It is known that an asymptotically nonexpansive mapping in the intermediate sense is not necessarily nonexpansive. Extending both [8, Theorem 1.1, 9, Theorem 5], the main result, Theorem 1, of this article provides a technical method to show the strong convergence of an iterative scheme to an element of $F(S) \cap V I(C, A)$, under the weaker assumption on the asymptotical nonexpansivity in the intermediate sense of $S$.

\section{Strong convergence theorems}

Let $C$ be a nonempty closed convex subset of a real Hilbert space $H$. For any $x$ in $H$, there exists a unique element in $C$, which is denoted by $P_{C} x$, such that $\left\|x-P_{C} x\right\| \leq \|$ $x-y \|$ for all $y$ in $C$. We call $P_{C}$ the metric projection of $H$ onto $C$. It is well-known that $P_{C}$ is a nonexpansive mapping from $H$ onto $C$, and

$$
\left\langle x-P_{C} x, P_{C} x-y\right\rangle \geq 0 \quad \text { for all } x \in H, y \in C ;
$$

see for example [10]. It is easy to see that (1) is equivalent to

$$
\|x-y\|^{2} \geq\left\|x-P_{C} x\right\|^{2}+\left\|y-P_{C} x\right\|^{2} \quad \text { for all } x \in H, y \in C .
$$

Let $A$ be a monotone mapping of $C$ into $H$. In the context of variational inequality problems, the characterization of the metric projection (1) implies that

$$
u \in \operatorname{VI}(C, A) \Leftrightarrow u=P_{C}(u-\lambda A u) \text { for some } \lambda>0 .
$$

Theorem 1. Let $C$ be a nonempty closed convex subset of a real Hilbert space H. Let $A: C \rightarrow H$ be a monotone and $k$-Lipschitz continuous mapping. Let $S: C \rightarrow C$ be a uniformly continuous asymptotically nonexpansive mapping in the intermediate sense with nonnegative null sequences $\left\{\gamma_{n}\right\}$ and $\left\{c_{n}\right\}$. Suppose that $\sum_{n=1}^{\infty} \lambda_{n}<\infty$ and $F(S) \cap V I$ $(C, A)$ is nonempty and bounded.

Assume that

(i) $0<\mu \leq 1$, and $0<a<b<\frac{3}{8 k \mu}$;

(ii) $a \leq \lambda_{n} \leq b, \alpha_{n} \geq 0, \beta_{n} \geq 0, \alpha_{n}+\beta_{n} \leq 1$, and $3 / 4<\delta_{n} \leq 1$, for all $n \geq 0$; 
(iii) $\lim _{n \rightarrow \infty} \alpha_{n}=0$;

(iv) $\lim _{\inf _{n \rightarrow \infty}} \beta_{n}>0$;

(v) $\lim _{n \rightarrow \infty} \beta_{n}=1$.

Set, for all $n \geq 0$,

$$
\begin{aligned}
\Delta_{n} & =\sup \left\{\left\|x_{n}-u\right\|: u \in F(S) \cap \operatorname{VI}(C, A)\right\}, \\
d_{n} & =2 b(1-\mu) \alpha_{n} \Delta_{n}, \\
w_{n} & =b^{2} \mu \alpha_{n}+4 b^{2} \mu^{2} \beta_{n}\left(1-\delta_{n}\right)\left(1+\gamma_{n}\right), \\
v_{n} & =b^{2}(1-\mu) \alpha_{n}+4 b^{2}(1-\mu)^{2} \beta_{n}\left(1-\delta_{n}\right)\left(1+\gamma_{n}\right), \text { and } \\
\vartheta_{n} & =\beta_{n} \gamma_{n} \Delta_{n}^{2}+\beta_{n} c_{n} .
\end{aligned}
$$

Let $\left\{x_{n}\right\},\left\{y_{n}\right\}$ and $\left\{z_{n}\right\}$ be sequences generated by the algorithm:

$$
\left\{\begin{array}{l}
x_{0} \in C \quad \text { chosen arbitrarily, } \\
y_{n}=\left(1-\delta_{n}\right) x_{n}+\delta_{n} P_{C}\left(x_{n}-\lambda_{n} \mu A x_{n}-\lambda_{n}(1-\mu) A y_{n}\right), \\
z_{n}=\left(1-\alpha_{n}-\beta_{n}\right) x_{n}+\alpha_{n} y_{n}+\beta_{n} S^{n} P_{C}\left(x_{n}-\lambda_{n} A y_{n}\right) \\
C_{n}=\left\{z \in C:\left\|z_{n}-z\right\|^{2} \leq\left\|x_{n}-z\right\|^{2}+d_{n}\left\|A y_{n}\right\|+w_{n}\left\|A x_{n}\right\|^{2}+v_{n}\left\|A y_{n}\right\|^{2}+\vartheta_{n}\right\} \\
Q_{n}=\left\{z \in C:\left\langle x_{n}-z_{1}, x_{0}-x_{n}\right\rangle \geq 0\right\}, \\
x_{n+1}=P_{C_{n} \cap Q_{n}}\left(x_{0}\right), \quad \forall n \geq 0 .
\end{array}\right.
$$

Then, the sequences $\left\{x_{n}\right\},\left\{y_{n}\right\}$ and $\left\{z_{n}\right\}$ in (3) are well-defined and converge strongly to the same point $q=P_{F(S) \cap V I(C, A)}\left(x_{0}\right)$.

Proof. First note that $\lim _{n \rightarrow \infty} \gamma_{n}=\lim _{n \rightarrow \infty} c_{n}=0$. We will see that $\left\{\Delta_{n}\right\}$ is bounded, and thus $\lim _{n \rightarrow \infty} d_{n}=\lim _{n \rightarrow \infty} w_{n}=\lim _{n \rightarrow \infty} v_{n}=\lim _{n \rightarrow \infty} \vartheta_{n}=0$.

We divide the proof into several steps.

Step 1. We claim that the following statements hold:

(a) $C_{n}$ is closed and convex for all $n \in \mathbb{N}$;

(b) $\left\|z_{n}-u\right\|^{2} \leq\left\|x_{n}-u\right\|^{2}+d_{n}\left\|A y_{n}\right\|+w_{n}\left\|A x_{n}\right\|^{2}+v_{n}\left\|A y_{n}\right\|^{2}+\vartheta_{n}$ for all $n \geq 0$ and $u \in F(S) \cap V I(C, A)$;

(c) $F(S) \cap V I(C, A) \subset C_{n}$ for all $n \in \mathbb{N}$.

It is obvious that $C_{n}$ is closed for all $n \in \mathbb{N}$. On the other hand, the defining inequality in $C_{n}$ is equivalent to the inequality

$$
\left\langle 2\left(x_{n}-z_{n}\right), z\right\rangle \leq\left\|x_{n}\right\|^{2}-\left\|z_{n}\right\|^{2}+d_{n}\left\|A y_{n}\right\|+w_{n}\left\|A x_{n}\right\|^{2}+v_{n}\left\|A y_{n}\right\|^{2}+\vartheta_{n}
$$

which is affine in $z$. Therefore, $C_{n}$ is convex.

Let $t_{n}=P_{C}\left(x_{n}-\lambda_{n} A y_{n}\right)$ for all $n \geq 0$. Assume that $u \in F(S) \cap V I(C, A)$ is arbitrary. In view of (3), the monotonicity of $A$, and the fact $u \in V I(C, A)$, we conclude that

$$
\begin{aligned}
& \left\|t_{n}-u\right\|^{2} \\
& \leq\left\|x_{n}-\lambda_{n} A y_{n}-u\right\|^{2}-\left\|x_{n}-\lambda_{n} A y_{n}-t_{n}\right\|^{2} \\
& =\left\|x_{n}-u\right\|^{2}-\left\|x_{n}-t_{n}\right\|^{2}+2 \lambda_{n}\left\langle A y_{n}, u-t_{n}\right\rangle \\
& =\left\|x_{n}-u\right\|^{2}-\left\|x_{n}-t_{n}\right\|^{2}+2 \lambda_{n}\left[\left\langle A y_{n}-A u, u-y_{n}\right\rangle+\left\langle A u, u-y_{n}\right\rangle+\left\langle A y_{n}, y_{n}-t_{n}\right\rangle\right] \\
& \leq\left\|x_{n}-u\right\|^{2}-\left\|x_{n}-t_{n}\right\|^{2}+2 \lambda_{n}\left\langle A y_{n}, y_{n}-t_{n}\right\rangle \\
& =\left\|x_{n}-u\right\|^{2}-\left\|x_{n}-y_{n}\right\|^{2}-2\left\langle x_{n}-y_{n}, y_{n}-t_{n}\right\rangle-\left\|y_{n}-t_{n}\right\|^{2}+2 \lambda_{n}\left\langle A y_{n}, y_{n}-t_{n}\right\rangle \\
& =\left\|x_{n}-u\right\|^{2}-\left\|x_{n}-y_{n}\right\|^{2}-\left\|y_{n}-t_{n}\right\|^{2}+2\left\langle x_{n}-\lambda_{n} A y_{n}-y_{n}, t_{n}-y_{n}\right\rangle .
\end{aligned}
$$


Now, using

$$
y_{n}=\left(1-\delta_{n}\right) x_{n}+\delta_{n} P_{C}\left(x_{n}-\lambda_{n} \mu A x_{n}-\lambda_{n}(1-\mu) A y_{n}\right),
$$

we estimate the last term

$$
\begin{aligned}
& \left\langle x_{n}-\lambda_{n} A y_{n}-y_{n}, t_{n}-y_{n}\right\rangle \\
= & \left\langle x_{n}-\lambda_{n} \mu A x_{n}-\lambda_{n}(1-\mu) A y_{n}-y_{n}, t_{n}-y_{n}\right\rangle+\lambda_{n} \mu\left\langle A x_{n}-A y_{n}, t_{n}-y_{n}\right\rangle \\
\leq \quad & \left\langle x_{n}-\lambda_{n} \mu A x_{n}-\lambda_{n}(1-\mu) A y_{n}-\left(1-\delta_{n}\right) x_{n}-\delta_{n} P_{C}\left(x_{n}-\lambda_{n} \mu A x_{n}-\lambda_{n}(1-\mu) A y_{n}\right), t_{n}-y_{n}\right\rangle \\
& \quad+\lambda_{n} \mu\left\|A x_{n}-A y_{n}\right\|\left\|t_{n}-y_{n}\right\| \\
\leq \quad & \delta_{n}\left\langle x_{n}-\lambda_{n} \mu A x_{n}-\lambda_{n}(1-\mu) A y_{n}-P_{C}\left(x_{n}-\lambda_{n} \mu A x_{n}-\lambda_{n}(1-\mu) A y_{n}\right), t_{n}-y_{n}\right\rangle \\
& \quad-\left(1-\delta_{n}\right) \lambda_{n}\left\langle\mu A x_{n}+(1-\mu) A y_{n}, t_{n}-y_{n}\right\rangle+\lambda_{n} \mu k\left\|x_{n}-y_{n}\right\|\left\|t_{n}-y_{n}\right\| .
\end{aligned}
$$

It follows from the properties (1) and (2) of the projection $P_{C}\left(x_{n}-\lambda_{n} \mu A x_{n}-\lambda_{n}(1-\mu)\right.$ $\left.A y_{n}\right)$ that

$$
\begin{array}{cc} 
& \left\langle x_{n}-\lambda_{n} \mu A x_{n}-\lambda_{n}(1-\mu) A y_{n}-P_{C}\left(x_{n}-\lambda_{n} \mu A x_{n}-\lambda_{n}(1-\mu) A y_{n}\right), t_{n}-y_{n}\right\rangle \\
= & \left\langle x_{n}-\lambda_{n} \mu A x_{n}-\lambda_{n}(1-\mu) A y_{n}-P_{C}\left(x_{n}-\lambda_{n} \mu A x_{n}-\lambda_{n}(1-\mu) A y_{n}\right),\right. \\
= & \left(1-\delta_{n}\right)\left\langle x_{n}-\lambda_{n} \mu A x_{n}-\lambda_{n}(1-\mu) A y_{n}-P_{C}\left(x_{n}-\lambda_{n} \mu A x_{n}-\lambda_{n}(1-\mu) A y_{n}\right), t_{n}-x_{n}\right\rangle \\
& +\delta_{n}\left\langle x_{n}-\lambda_{n} \mu A x_{n}-\lambda_{n}(1-\mu) A y_{n}-P_{C}\left(x_{n}-\lambda_{n} \mu A x_{n}-\lambda_{n}(1-\mu) A y_{n}\right),\right. \\
& \left.t_{n}-P_{C}\left(x_{n}-\lambda_{n} \mu A x_{n}-\lambda_{n}(1-\mu) A y_{n}\right)\right\rangle \\
\leq & \left(1-\delta_{n}\right)\left\langle x_{n}-\lambda_{n} \mu A x_{n}-\lambda_{n}(1-\mu) A y_{n}-P_{C}\left(x_{n}-\lambda_{n} \mu A x_{n}-\lambda_{n}(1-\mu) A y_{n}\right), t_{n}-x_{n}\right\rangle \\
\leq & \left(1-\delta_{n}\right) \| x_{n}-\lambda_{n} \mu A x_{n}-\lambda_{n}(1-\mu) A y_{n}-P_{C}\left(x_{n}-\lambda_{n} \mu A x_{n}-\lambda_{n}(1-\mu) A y_{n}\|\| t_{n}-x_{n} \|\right. \\
\leq & \left(1-\delta_{n}\right)\left\|\lambda_{n} \mu A x_{n}+\lambda_{n}(1-\mu) A y_{n}\right\|\left\|t_{n}-x_{n}\right\| \\
\leq & \left(1-\delta_{n}\right) \lambda_{n}\left(\mu\left\|A x_{n}\right\|+(1-\mu)\left\|A y_{n}\right\|\right)\left(\left\|t_{n}-y_{n}\right\|+\left\|y_{n}-x_{n}\right\|\right) .
\end{array}
$$

In view of (4)-(6), $\lambda_{n} \leq b$, and the inequalities $2 \alpha \beta \leq \alpha^{2}+\beta^{2}$ and $(\alpha+\beta)^{2} \leq 2 \alpha^{2}+$ $2 \beta^{2}$, we conclude that

$$
\begin{aligned}
\left\|t_{n}-u\right\|^{2} \leq & \left\|x_{n}-u\right\|^{2}-\left\|x_{n}-y_{n}\right\|^{2}-\left\|y_{n}-t_{n}\right\|^{2}+2\left\langle x_{n}-\lambda_{n} A y_{n}-y_{n}, t_{n}-y_{n}\right\rangle \\
\leq & \left\|x_{n}-u\right\|^{2}-\left\|x_{n}-y_{n}\right\|^{2}-\left\|y_{n}-t_{n}\right\|^{2} \\
& +2 \lambda_{n}\left[\delta_{n}\left(1-\delta_{n}\right)\left(\mu\left\|A x_{n}\right\|+(1-\mu)\left\|A y_{n}\right\|\right)\left(\left\|t_{n}-y_{n}\right\|+\left\|y_{n}-x_{n}\right\|\right)\right. \\
& \left.\quad-2\left(1-\delta_{n}\right) \lambda_{n}\left\langle\mu A x_{n}+(1-\mu) A y_{n}, t_{n}-y_{n}\right\rangle+2 \lambda_{n} \mu k\left\|x_{n}-y_{n}\right\|\left\|t_{n}-y_{n}\right\|\right] \\
\leq & \left\|x_{n}-u\right\|^{2}-\left\|x_{n}-y_{n}\right\|^{2}-\left\|y_{n}-t_{n}\right\|^{2} \\
& +2 \delta_{n}\left(1-\delta_{n}\right) b\left(\mu\left\|A x_{n}\right\|+(1-\mu)\left\|A y_{n}\right\|\right)\left(\left\|t_{n}-y_{n}\right\|+\left\|y_{n}-x_{n}\right\|\right) \\
& +2\left(1-\delta_{n}\right) b\left(\mu\left\|A x_{n}\right\|+(1-\mu)\left\|A y_{n}\right\|\right)\left\|t_{n}-y_{n}\right\|+2 b \mu k\left\|x_{n}-y_{n}\right\|\left\|t_{n}-y_{n}\right\| \\
= & \left\|x_{n}-u\right\|^{2}-\left\|x_{n}-y_{n}\right\|^{2}-\left\|y_{n}-t_{n}\right\|^{2} \\
& +2 \delta_{n}\left(1-\delta_{n}\right)\left(b^{2} \mu^{2}\left\|A x_{n}\right\|^{2}+b^{2}(1-\mu)^{2}\left\|A y_{n}\right\|^{2}+\left\|t_{n}-y_{n}\right\|^{2}+\left\|y_{n}-x_{n}\right\|^{2}\right) \\
& +\left(1-\delta_{n}\right)\left(b^{2} \mu^{2}\left\|A x_{n}\right\|^{2}+b^{2}(1-\mu)^{2}\left\|A y_{n}\right\|^{2}+2\left\|t_{n}-y_{n}\right\|^{2}\right) \\
& +b \mu k\left(\left\|x_{n}-y_{n}\right\|^{2}+\left\|t_{n}-y_{n}\right\|^{2}\right) \\
= & \left\|x_{n}-u\right\|^{2}-\left\|x_{n}-y_{n}\right\|^{2}\left(1-2 \delta_{n}\left(1-\delta_{n}\right)-b k \mu\right) \\
& -\left\|t_{n}-y_{n}\right\|^{2}\left(2 \delta_{n}^{2}-\delta_{n}-b k \mu\right) \\
& +2\left(1-\delta_{n}^{2}\right) b^{2} \mu^{2}\left\|A x_{n}\right\|^{2}+2\left(1-\delta_{n}^{2}\right) b^{2}(1-\mu)^{2}\left\|A y_{n}\right\|^{2} .
\end{aligned}
$$

Since $\frac{3}{4}<\delta_{n} \leq 1$ and $b<\frac{3}{8 k \mu}$, we have from (7) for all $n \in \mathbb{N}$,

$$
\left\|t_{n}-u\right\|^{2} \leq\left\|x_{n}-u\right\|^{2}+4\left(1-\delta_{n}\right) b^{2} \mu^{2}\left\|A x_{n}\right\|^{2}+4\left(1-\delta_{n}\right) b^{2}(1-\mu)^{2}\left\|A y_{n}\right\|^{2} .
$$


In view of the fact that $u \in V I(A, C)$ and properties of $P_{C}$, we obtain

$$
\begin{aligned}
\left\|y_{n}-u\right\|^{2}= & \left\|\left(1-\delta_{n}\right)\left(x_{n}-u\right)+\delta_{n}\left(P_{C}\left(x_{n}-\lambda_{n} \mu A x_{n}-\lambda_{n}(1-\mu) A y_{n}\right)-u\right)\right\|^{2} \\
\leq & \left(1-\delta_{n}\right)\left\|x_{n}-u\right\|^{2}+\delta_{n}\left\|P_{C}\left(x_{n}-\lambda_{n} \mu A x_{n}-\lambda_{n}(1-\mu) A y_{n}\right)-P_{C}(u)\right\|^{2} \\
\leq & \left(1-\delta_{n}\right)\left\|x_{n}-u\right\|^{2}+\delta_{n}\left\|x_{n}-\lambda_{n} \mu A x_{n}-\lambda_{n}(1-\mu) A y_{n}-u\right\|^{2} \\
= & \left(1-\delta_{n}\right)\left\|x_{n}-u\right\|^{2}+\delta_{n}\left[\left\|x_{n}-u\right\|^{2}-2\left\langle\lambda_{n} \mu A x_{n}+\lambda_{n}(1-\mu) A y_{n}, x_{n}-u\right\rangle\right. \\
& \left.+\left\|\lambda_{n} \mu A x_{n}+\lambda_{n}(1-\mu) A y_{n}\right\|^{2}\right] \\
= & \left(1-\delta_{n}\right)\left\|x_{n}-u\right\|^{2}+\delta_{n}\left[\left\|x_{n}-u\right\|^{2}-2 \lambda_{n} \mu\left\langle A x_{n}, x_{n}-u\right\rangle-2 \lambda_{n}(1-\mu)\left\langle A y_{n}, x_{n}-u\right\rangle\right. \\
& \left.+\left\|\lambda_{n} \mu A x_{n}+\lambda_{n}(1-\mu) A y_{n}\right\|^{2}\right] \\
\leq & \left(1-\delta_{n}\right)\left\|x_{n}-u\right\|^{2}+\delta_{n}\left[\left\|x_{n}-u\right\|^{2}+2 \lambda_{n}(1-\mu)\left\|A y_{n}\right\|\left\|x_{n}-u\right\|\right. \\
& \left.+\lambda_{n}^{2} \mu\left\|A x_{n}\right\|^{2}+\lambda_{n}^{2}(1-\mu)\left\|A y_{n}\right\|^{2}\right] \\
\leq & \left(1-\delta_{n}\right)\left\|x_{n}-u\right\|^{2}+\delta_{n}\left[\left\|x_{n}-u\right\|^{2}+2 b(1-\mu) \Delta_{n}\left\|A y_{n}\right\|\right. \\
& \left.+b^{2} \mu\left\|A x_{n}\right\|^{2}+b^{2}(1-\mu)\left\|A y_{n}\right\|^{2}\right] \\
\leq & \left\|x_{n}-u\right\|^{2}+\delta_{n}\left[2 b(1-\mu) \Delta_{n}\left\|A y_{n}\right\|+b^{2} \mu^{2}\left\|A x_{n}\right\|^{2}+b^{2}(1-\mu)^{2}\left\|A y_{n}\right\|^{2}\right] \\
\leq & \left\|x_{n}-u\right\|^{2}+2 b(1-\mu) \Delta_{n}\left\|A y_{n}\right\|+b^{2} \mu\left\|A x_{n}\right\|^{2}+b^{2}(1-\mu)\left\|A y_{n}\right\|^{2} .
\end{aligned}
$$

Since $S$ is asymptotically nonexpansive in the intermediate sense, in view of $S^{n} u=u$, we conclude that

$$
\begin{aligned}
\left\|z_{n}-u\right\|^{2}= & \left\|\left(1-\alpha_{n}-\beta_{n}\right) x_{n}+\alpha_{n} y_{n}+\beta_{n} S^{n} t_{n}-u\right\|^{2} \\
\leq & \left(1-\alpha_{n}-\beta_{n}\right)\left\|x_{n}-u\right\|^{2}+\alpha_{n}\left\|y_{n}-u\right\|^{2}+\beta_{n}\left\|S^{n} t_{n}-u\right\|^{2} \\
\leq & \left(1-\alpha_{n}-\beta_{n}\right)\left\|x_{n}-u\right\|^{2} \\
& +\alpha_{n}\left[\left\|x_{n}-u\right\|^{2}+2 b(1-\mu) \Delta_{n}\left\|A y_{n}\right\|+b^{2} \mu\left\|A x_{n}\right\|^{2}+b^{2}(1-\mu)\left\|A y_{n}\right\|^{2}\right] \\
& +\beta_{n}\left[\left(1+\gamma_{n}\right)\left\|t_{n}-u\right\|^{2}+c_{n}\right] \\
\leq & \left(1-\alpha_{n}-\beta_{n}\right)\left\|x_{n}-u\right\|^{2} \\
& +\alpha_{n}\left[\left\|x_{n}-u\right\|^{2}+2 b(1-\mu) \Delta_{n}\left\|A y_{n}\right\|+b^{2} \mu\left\|A x_{n}\right\|^{2}+b^{2}(1-\mu)\left\|A y_{n}\right\|^{2}\right] \\
& +\beta_{n}\left(1+\gamma_{n}\right)\left[\left\|x_{n}-u\right\|^{2}+2\left(1-\delta_{n}\right) b^{2} \mu^{2}\left\|A x_{n}\right\|^{2}+2\left(1-\delta_{n}\right) b^{2}(1-\mu)^{2}\left\|A y_{n}\right\|^{2}\right] \\
& +\beta_{n} c_{n} \\
\leq & \left\|x_{n}-u\right\|^{2}+\beta_{n} \gamma_{n} \Delta_{n}^{2}+2 b(1-\mu) \alpha_{n} \Delta_{n}\left\|A y_{n}\right\| \\
& +\left(b^{2} \mu \alpha_{n}+2 b^{2} \mu^{2} \beta_{n}\left(1-\delta_{n}\right)\left(1+\gamma_{n}\right)\right)\left\|A x_{n}\right\|^{2} \\
& +\left(b^{2}(1-\mu) \alpha_{n}+2 b^{2}(1-\mu)^{2} \beta_{n}\left(1-\delta_{n}\right)\left(1+\gamma_{n}\right)\right)\left\|A y_{n}\right\|^{2} \\
& +\beta_{n} c_{n} .
\end{aligned}
$$

This implies that $u \in C_{n}$. Therefore, $F(S) \cap V I(C, A) \subset C_{n}$.

Step 2. We prove that the sequence $\left\{x_{n}\right\}$ is well-defined and $F(S) \cap V I(C, A) \subset C_{n} \cap$ $Q_{n}$ for all $n \geq 0$.

We prove this assertion by mathematical induction. For $n=0$ we get $Q_{0}=C$. Hence, by step 1 , we deduce that $F(S) \cap V I(C, A) \subset C_{1} \cap Q_{1}$. Assume that $x_{k}$ is defined and $F$ $(S) \cap V I(C, A) \subset C_{k} \cap Q_{k}$ for some $k \geq 1$. Then, $y_{k}, z_{k}$ are well-defined elements of $C$. We notice that $C_{k}$ is a closed convex subset of $C$ since

$$
C_{k}=\left\{z \in C:\left\|z_{k}-x_{k}\right\|^{2}+2\left\langle z_{k}-x_{k}, x_{k}-z\right\rangle \leq d_{n}\left\|A y_{n}\right\|+w_{n}\left\|A x_{n}\right\|^{2}+v_{n}\left\|A y_{n}\right\|^{2}+\vartheta_{n}\right\}
$$

It is easy to see that $Q_{k}$ is closed and convex. Therefore, $C_{k} \cap Q_{k}$ is a closed and convex subset of $C$, since by the assumption we have $F(S) \cap V I(C, A) \subset C_{k} \cap Q_{k}$. This means that $P_{C_{k} \cap Q_{k}} x_{0}$ is well-defined.

By the definition of $x_{k+1}$ and of $Q_{k+1}$, we deduce that $C_{k} \cap Q_{k} \subset Q_{k+1}$. Hence, $F(S) \cap$ $V I(C, A) \subset Q_{k+1}$. Exploiting Step 1 we conclude that $F(S) \cap V I(C, A) \subset C_{k+1} \cap Q_{k+1}$. 
Step 3. We claim that the following assertions hold:

(d) $\lim _{n \rightarrow \infty}\left\|x_{n}-x_{0}\right\|$ exists and hence $\left\{x_{n}\right\}$, as well as $\left\{\Delta_{n}\right\}$, is bounded.

(e) $\lim _{\mathrm{n} \rightarrow \infty}|| x_{n+1}-x_{n} \|=0$.

(f) $\lim _{\mathrm{n} \rightarrow \infty}|| z_{n}-x_{n} \|=0$.

Let $u \in F(S) \cap V I(C, A)$. Since $x_{n+1}=P_{C_{n} \cap Q_{n}} x_{0}$ and $u \in F(S) \cap V I(C, A) \subset C_{n} \cap Q_{n}$, we conclude that

$$
\left\|x_{n+1}-x_{0}\right\| \leq\left\|u-x_{0}\right\|, \quad \forall n \geq 0 .
$$

This means that $\left\{x_{n}\right\}$ is bounded, and so are $\left\{y_{n}\right\}, A x_{n}$ and $\left\{A y_{n}\right\}$, because of the Lipschitz-continuity of $A$. On the other hand, we have $x_{n}=P_{Q_{n}} x_{0}$ and $x_{n+1} \in C_{n} \cap Q_{n}$ $\subset Q_{n}$. This implies that

$$
\left\|x_{n+1}-x_{n}\right\|^{2} \leq\left\|x_{n+1}-x_{0}\right\|^{2}-\left\|x_{n}-x_{0}\right\|^{2}, \quad \forall n \geq 0 .
$$

In particular, $\left\|x_{n+1}-x_{0}\right\| \geq\left\|x_{n}-x_{0}\right\|$ hence $\lim _{n \rightarrow \infty}\left\|x_{n}-x_{0}\right\|$ exists. It follows from (12) that

$$
\lim _{n \rightarrow \infty}\left(x_{n+1}-x_{n}\right)=0 .
$$

Since $x_{n+1} \in C_{n}$, we obtain

$$
\left\|z_{n}-x_{n+1}\right\|^{2} \leq\left\|x_{n}-x_{n+1}\right\|^{2}+d_{n}\left\|A y_{n}\right\|+w_{n}\left\|A x_{n}\right\|^{2}+v_{n}\left\|A y_{n}\right\|^{2}+\vartheta_{n} .
$$

In view of $\lim _{n \rightarrow \infty} \gamma_{n}=0, \lim _{n \rightarrow \infty} \alpha_{n}=0, \lim _{n \rightarrow \infty} \delta_{n}=1$ and from the boundedness of $\left\{A x_{n}\right\}$ and $\left\{A y_{n}\right\}$ we infer that $\lim _{n \rightarrow \infty}\left(x_{n+1}-z_{n}\right)=0$. Combining with (13) we deduce that $\lim _{n \rightarrow \infty}\left(x_{n}-z_{n}\right)=0$.

Step 4. We claim that the following assertions hold:

(g) $\lim _{n \rightarrow \infty}|| x_{n}-y_{n} \|=0$.

(h) $\lim _{n \rightarrow \infty}\left\|S x_{n}-x_{n}\right\|=0$.

In view of (3), $z_{n}=\left(1-\alpha_{n}-\beta_{n}\right) x_{n}+\alpha_{n} y_{n}+\beta_{n} S^{n} t_{n}$, and $S^{n} u=u$, we obtain from (9) and (8) that

$$
\begin{aligned}
& \left\|z_{n}-u\right\|^{2} \\
= & \left\|\left(1-\alpha_{n}-\beta_{n}\right) x_{n}+\alpha_{n} y_{n}+\beta_{n} S^{n} t_{n}-u\right\|^{2} \\
\leq & \left(1-\alpha_{n}-\beta_{n}\right)\left\|x_{n}-u\right\|^{2}+\alpha_{n}\left\|y_{n}-u\right\|^{2}+\beta_{n}\left\|S^{n} t_{n}-u\right\|^{2} \\
\leq & \left(1-\alpha_{n}-\beta_{n}\right)\left\|x_{n}-u\right\|^{2} \\
& +\alpha_{n}\left[\left\|x_{n}-u\right\|^{2}+2 b(1-\mu) \Delta_{n}\left\|A y_{n}\right\|+b^{2} \mu\left\|A x_{n}\right\|^{2}+b^{2}(1-\mu)\left\|A y_{n}\right\|^{2}\right] \\
& +\beta_{n}\left[\left(1+\gamma_{n}\right)\left\|t_{n}-u\right\|^{2}+c_{n}\right] \\
\leq & \left(1-\alpha_{n}-\beta_{n}\right)\left\|x_{n}-u\right\|^{2} \\
& +\alpha_{n}\left[\left\|x_{n}-u\right\|^{2}+2 b(1-\mu) \Delta_{n}\left\|A y_{n}\right\|+b^{2} \mu\left\|A x_{n}\right\|^{2}(1-\mu)\left\|A y_{n}\right\|^{2}\right] \\
& +\beta_{n}\left(1+\gamma_{n}\right)\left[\left\|x_{n}-u\right\|^{2}-\left(1-2 \delta_{n}\left(1-\delta_{n}\right)\left\|x_{n}-\gamma_{n}\right\|^{2}-b k \mu\right)\right. \\
& \quad-\left(2 \delta_{n}^{2}-1-b k \mu\right)\left\|t_{n}-\gamma_{n}\right\|^{2}+4\left(1-\delta_{n}\right) b^{2} \mu^{2}\left\|A x_{n}\right\|^{2} \\
& \quad+4\left(1-\delta_{n}\right) b^{2}(1-\mu)^{2}\left\|A y_{n}\right\|^{2}+\beta_{n} c_{n} \\
\leq & \left\|x_{n}-u\right\|^{2}+\beta_{n} \gamma_{n} \Delta_{n}^{2}+\beta_{n} c_{n} \\
& +2 b(1-\mu) \alpha_{n} \Delta_{n}\left\|A y_{n}\right\|+\left[b^{2} \mu \alpha_{n}+4 b^{2} \mu^{2} \beta_{n}\left(1+\gamma_{n}\right)\left(1-\delta_{n}\right)\right]\left\|A x_{n}\right\|^{2} \\
& +\left[b^{2}(1-\mu) \alpha_{n}+4 b^{2}(1-\mu)^{2} \beta_{n}\left(1+\gamma_{n}\right)\left(1-\delta_{n}\right)\right]\left\|A y_{n}\right\|^{2} \\
& -\left[\beta_{n}\left(1+\gamma_{n}\right)\left(1-2 \delta_{n}\left(1-\delta_{n}\right)-b k \mu\right)\right]\left\|x_{n}-\gamma_{n}\right\|^{2} \\
& -\left[\beta_{n}\left(1+\gamma_{n}\right)\left(2 \delta_{n}^{2}-\delta_{n}-b k \mu\right)\right]\left\|t_{n}-\gamma_{n}\right\|^{2} .
\end{aligned}
$$


Thus, we have

$$
\begin{aligned}
& \beta_{n}\left(1+\gamma_{n}\right)\left(1-2 \delta_{n}\left(1-\delta_{n}\right)-b k \mu\right)\left\|x_{n}-\gamma_{n}\right\|^{2} \\
\leq & \left\|x_{n}-u\right\|^{2}-\left\|z_{n}-u\right\|^{2}+\beta_{n} \gamma_{n} \Delta_{n}^{2}+\beta_{n} c_{n} \\
& +2 b(1-\mu) \alpha_{n} \Delta_{n}\left\|A y_{n}\right\|+\left[b^{2} \mu \alpha_{n}+4 b^{2} \mu^{2} \beta_{n}\left(1+\gamma_{n}\right)\left(1-\delta_{n}\right)\right]\left\|A x_{n}\right\|^{2} \\
& +\left[b^{2}(1-\mu) \alpha_{n}+4 b^{2}(1-\mu)^{2} \beta_{n}\left(1+\gamma_{n}\right)\left(1-\delta_{n}\right)\right]\left\|A y_{n}\right\|^{2} \\
\leq & \left(\left\|x_{n}-u\right\|+\left\|z_{n}-u\right\|\right)\left\|x_{n}-z_{n}\right\|+\beta_{n} \gamma_{n} \Delta_{n}^{2}+\beta_{n} c_{n} \\
& +2 b(1-\mu) \alpha_{n} \Delta_{n}\left\|A y_{n}\right\|+\left[b^{2} \mu \alpha_{n}+4 b^{2} \mu^{2} \beta_{n}\left(1+\gamma_{n}\right)\left(1-\delta_{n}\right)\right]\left\|A x_{n}\right\|^{2} \\
& +\left[b^{2}(1-\mu) \alpha_{n}+4 b^{2}(1-\mu)^{2} \beta_{n}\left(1+\gamma_{n}\right)\left(1-\delta_{n}\right)\right]\left\|A y_{n}\right\|^{2} .
\end{aligned}
$$

Since $b k \mu<3 / 8$ and $3 / 4 \leq \delta_{n} \leq 1$ for all $n \geq 0$, we have

$$
\lim _{n \rightarrow \infty}\left\|x_{n}-y_{n}\right\|^{2}=0 .
$$

In the same manner, from (14), we conclude that

$$
\lim _{n \rightarrow \infty}\left\|t_{n}-y_{n}\right\|^{2}=0
$$

Since $A$ is $k$-Lipschitz continuous, we obtain $\left\|A y_{n}-A x_{n}\right\| \rightarrow 0$. On the other hand,

$$
\left\|x_{n}-t_{n}\right\| \leq\left\|x_{n}-y_{n}\right\|+\left\|y_{n}-t_{n}\right\|,
$$

which implies that $\left\|x_{n}-t_{n}\right\| \rightarrow 0$. Since $z_{n}=\left(1-\alpha_{n}-\beta_{n}\right) x_{n}+\alpha_{n} y_{n}+\beta_{n} S^{n} t_{n}$, we have

$$
z_{n}-x_{n}=-\alpha_{n} x_{n}+\alpha_{n} y_{n}+\beta_{n}\left(s^{n} t_{n}-x_{n}\right) .
$$

From $\left\|z_{n}-x_{n}\right\| \rightarrow 0, \alpha_{n} \rightarrow 0, \lim _{n \rightarrow 0} \beta_{n}>0$ and the boundedness of $\left\{x_{n}, y_{n}\right\}$ we deduce that $\left\|S^{n} t_{n}-x_{n}\right\| \rightarrow 0$. Thus, we get $\left\|t_{n}-S^{n} t_{n}\right\| \rightarrow 0$. By the triangle inequality, we obtain

$$
\begin{aligned}
\left\|x_{n}-S^{n} x_{n}\right\| & \leq\left\|x_{n}-t_{n}\right\|+\left\|t_{n}-S^{n} t_{n}\right\|+\left\|S^{n} t_{n}-S^{n} x_{n}\right\| \\
& \leq\left\|x_{n}-t_{n}\right\|+\left\|t_{n}-S^{n} t_{n}\right\|+\sqrt{\left(1+\gamma_{n}\right)\left\|t_{n}-x_{n}\right\|+c_{n}} .
\end{aligned}
$$

Hence, $\left\|x_{n}-S^{n} x_{n}\right\| \rightarrow 0$. Since $\left\|x_{n}-x_{n+1}\right\| \rightarrow 0$, it follows from Lemma 2.7 of Sahu et al. [5] that $\left\|x_{n}-S x_{n}\right\| \rightarrow 0$. By the uniform continuity of $S$, we obtain $\left\|x_{n}-S^{m} x_{n}\right\|$ $\rightarrow 0$ as $n \rightarrow \infty$ for all $m \geq 1$.

Step 5. We claim that $\omega_{w}\left(x_{n}\right) \subset F(S) \cap V I(C, A)$, where

$$
\omega_{w}\left(x_{n}\right):=\left\{x \in H: x_{n_{j}} \rightarrow x \text { weakly for some subsequence }\left\{x_{n_{j}}\right\} \text { of }\left\{x_{n}\right\}\right\} .
$$

The proof of this step is similar to that of [8, Theorem 1.1, step 5] and we omit it.

A similar argument as mentioned in [9, Theorem 5, Step 6] proves the following assertion.

Step 6. The sequences $\left\{x_{n}\right\},\left\{y_{n}\right\}$ and $\left\{z_{n}\right\}$ converge strongly to the same point $q=P_{F}$ $(S) \cap V I(C, A)\left(x_{0}\right)$, which completes the proof.

For $\alpha_{n}=0, \beta_{n}=1$ and $\delta_{n}=1$ for all $n \in \mathbb{N}$ in Theorem 1, we get the following corollary.

Corollary 2. Let $C$ be a nonempty closed convex subset of a real Hilbert spaces $H$. Let $A: C \rightarrow H$ be a monotone and $k$-Lipschitz continuous mapping and let $S: C \rightarrow C$ be a 
uniformly continuous asymptotically nonexpansive mapping in the intermediate sense with nonnegative null sequences $\left\{\gamma_{n}\right\}$ and $\left\{c_{n}\right\}$.

Suppose that $\sum_{n=1}^{\infty} \gamma_{n}<\infty$ and $F(S) \cap \operatorname{VI}(C, A)$ is nonempty and bounded. Set $\vartheta_{n}=$ $\gamma_{n} \Delta_{n}+c_{n}$. Let $\mu$ be a constant in $(0,1]$, and let $\left\{\lambda_{n}\right\}$ be a sequence in $[a, b]$ with $a>0$ and $b<\frac{3}{8 k \mu}$.

Let $\left\{x_{n}\right\},\left\{y_{n}\right\}$ and $\left\{z_{n}\right\}$ be sequences generated by

$$
\left\{\begin{array}{l}
x_{0} \in C \quad \text { chosen arbitrarily, } \\
y_{n}=P_{C}\left(x_{n}-\lambda_{n} \mu A x_{n}-\lambda_{n}(1-\mu) A y_{n}\right), \\
z_{n}=S^{n} P_{C}\left(x_{n}-\lambda_{n} A y_{n}\right) \\
C_{n}=\left\{z \in C:\left\|z_{n}-z\right\|^{2} \leq\left\|x_{n}-z\right\|^{2}+\vartheta_{n}\right\} \\
Q_{n}=\left\{z \in C:\left\langle x_{n}-z, x_{0}-x_{n}\right\rangle \geq 0\right\} \\
x_{n+1}=P_{C_{n} \cap Q_{n}}\left(x_{0}\right), \quad \forall n \geq 0 .
\end{array}\right.
$$

Then, the sequences $\left\{x_{n}\right\},\left\{y_{n}\right\}$ and $\left\{z_{n}\right\}$ in (15) are well-defined and converge strongly to the same point $q=P_{F(S) \cap V I(C, A)}\left(x_{0}\right)$.

In Theorem 1, if we set $\alpha_{n}=0$ and $\beta_{n}=1$ for all $n \in \mathbb{N}$ then the following result concerning variational inequality problems holds.

Corollary 3. Let $C$ be a nonempty closed convex subset of a real Hilbert spaces $H$. Let $A: C \rightarrow H$ be a monotone and $k$-Lipschitz continuous mapping and let $S: C \rightarrow C$ be a uniformly continuous asymptotically nonexpansive mapping in the intermediate sense with null sequences $\left\{\gamma_{n}\right\}$ and $\left\{c_{n}\right\}$.

Suppose that $\sum_{n=1}^{\infty} \gamma_{n}<\infty$ and $F(S) \cap \operatorname{VI}(C, A)$ is nonempty and bounded. Let $\mu$ be a constant in $(0,1]$, let $\left\{\lambda_{n}\right\}$ be a sequence in $[\mathrm{a}, \mathrm{b}]$ with $a>0$ and $b<\frac{3}{8 k \mu}$, and let $\left\{\delta_{n}\right\}$ be a sequence in $[0,1]$ such that $\lim _{n \rightarrow \infty} \delta_{n}=1$ and $\delta_{n}>\frac{3}{4}$ for all $n \geq 0$. Set $\Delta_{n}=\sup$ $\left\{\left\|x_{n}-u\right\|: u \in F(S) \cap V I(C, A)\right\}, w_{n}=4 b^{2} \mu^{2}\left(1+\gamma_{n}\right)\left(1-\delta_{n}\right), \vartheta_{n}=\gamma_{n} \Delta_{n}+c_{n}$ for all $n \geq$ 0 .

Let $\left\{x_{n}\right\},\left\{y_{n}\right\}$ and $\left\{z_{n}\right\}$ be sequences generated by

$$
\left\{\begin{array}{l}
x_{0} \in C \quad \text { chosen arbitrarily, } \\
y_{n}=\left(1-\delta_{n}\right) x_{n}+\delta_{n} P_{C}\left(x_{n}-\lambda_{n} \mu A x_{n}-\lambda_{n}(1-\mu) A y_{n}\right), \\
z_{n}=S^{n} P_{C}\left(x_{n}-\lambda_{n} A y_{n}\right), \\
C_{n}=\left\{z \in C:\left\|z_{n}-z\right\|^{2} \leq\left\|x_{n}-z\right\|^{2}+w_{n}\left\|A x_{n}\right\|^{2}+\vartheta_{n}\right\}, \\
Q_{n}=\left\{z \in C:\left\langle x_{n}-z, x_{0}-x_{n}\right\rangle \geq 0\right\}, \\
x_{n+1}=P_{C_{n} \cap Q_{n}}\left(x_{0}\right), \quad \forall n \geq 0 .
\end{array}\right.
$$

Then, the sequences $\left\{x_{n}\right\},\left\{y_{n}\right\}$ and $\left\{z_{n}\right\}$ in (16) are well-defined and converge strongly to the same point $q=P_{F(s) \cap V I(C, A)}\left(x_{0}\right)$.

The following theorem is yet an other easy consequence of Theorem 1.

Corollary 4. Let $H$ be a real Hilbert space. Let $A: H \rightarrow H$ be a monotone and $k$ Lipschitz continuous mapping and let $S: H \rightarrow H$ be a uniformly continuous asymptotically nonexpan-sive mapping in the intermediate sense with null sequences $\left\{\gamma_{n}\right\}$ and $\left\{c_{n}\right\}$.

Suppose that $\sum_{n=1}^{\infty} \gamma_{n}<\infty$ and $F(S) \cap A^{-1}(0)$ is nonempty and bounded. Let $\mu$ be a constant in $(0,1]$, let $\left\{\lambda_{n}\right\}$ be a sequence in $[a, 3 b / 4]$ with $0<4 a / 3<b<\frac{3}{8 k \mu}$, and let $\left\{\alpha_{n}\right\},\left\{\beta_{n}\right\}$ and $\left\{\delta_{n}\right\}$ be three sequences in $[0,1]$ satisfying the following conditions: 
(i) $\alpha_{n}+\beta_{n} \leq 1, \forall n \geq 0$;

(ii) $\lim _{n \rightarrow \infty} \alpha_{n}=0$;

(iii) $\lim _{\inf _{n \rightarrow \infty}} \beta_{n}>0$;

(iv) $\lim _{n \rightarrow \infty} \delta_{n}=1$ and $\delta_{n}>\frac{3}{4}$ for all $n \geq 0$.

Set

$$
\begin{aligned}
\Delta_{n} & =\sup \left\{\left\|x_{n}-u\right\|: u \in F(S) \cap A^{-1}(0)\right\}, \\
d_{n} & =2 b(1-\mu) \alpha_{n} \Delta_{n}, \\
w_{n} & =b^{2} \mu \alpha_{n}+4 b^{2} \mu^{2} \beta_{n}\left(1-\delta_{n}\right)\left(1+\gamma_{n}\right), \\
v_{n} & =b^{2}(1-\mu) \alpha_{n}+4 b^{2}(1-\mu)^{2} \beta_{n}\left(1-\delta_{n}\right)\left(1+\gamma_{n}\right), \text { and } \\
\vartheta_{n} & =\beta_{n} \gamma_{n} \Delta_{n}^{2}+\beta_{n} c_{n}
\end{aligned}
$$

for all $n \geq 0$.

Let $\left\{x_{n}\right\},\left\{y_{n}\right\}$ and $\left\{z_{n}\right\}$ be sequences generated by

$$
\left\{\begin{array}{l}
x_{0} \in C \quad \text { chosen arbitrarily, } \\
y_{n}=x_{n}-\lambda_{n} \mu A x_{n}-\lambda_{n}(1-\mu) A y_{n}, \\
z_{n}=\left(1-\beta_{n}\right) x_{n}-\alpha_{n} \mu A x_{n}-\alpha_{n} \lambda_{n}(1-\mu) A y_{n}+\beta_{n} S^{n}\left(x_{n}-\frac{\lambda_{n}}{\delta_{n}} A y_{n}\right), \\
C_{n}=\left\{z \in C:\left\|z_{n}-z\right\|^{2} \leq\left\|x_{n}-z\right\|^{2}+d n\left\|A y_{n}\right\|+w_{n}\left\|A x_{n}\right\|^{2}+v_{n}\left\|A y_{n}\right\|^{2}+\vartheta_{n}\right\} \\
Q_{n}=\left\{z \in C:\left\langle x_{n}-z, x_{0}-x_{n}\right\rangle \geq 0\right\}, \\
x_{n+1}=P_{C_{n} \cap Q_{n}}\left(x_{0}\right), \quad \forall n \geq 0 .
\end{array}\right.
$$

Then, the sequences $\left\{x_{n}\right\},\left\{y_{n}\right\}$ and $\left\{z_{n}\right\}$ in (17) are well-defined and converge strongly to the same point $q=P_{F(S) \cap A^{-1}}(0)\left(x_{0}\right)$.

Proof. Replace $\lambda_{n}$ by $\lambda_{n}^{\prime}=\frac{\lambda_{n}}{\delta_{n}}$. Then, $a \leq \lambda_{n}^{\prime}<\frac{4}{3} \lambda_{n}<b<\frac{3}{8 k \mu}$. For $C=H$, we have $P_{C}$ $=I$ and $V I(C, A)=A^{-1}(0)$. In view of Theorem 1, the sequences $\left\{x_{n}\right\},\left\{y_{n}\right\}$ and $\left\{z_{n}\right\}$ are well-defined and converge strongly to the same point $q=P_{F(S) \cap A^{-1}}(0)\left(x_{0}\right)$.

\section{Acknowledgements}

The first author of this study was conducted with a postdoctoral fellowship at the National Sun Yat-Sen University, Kaohsiung 804, Taiwan. The second and third authors of this research were partially supported by the Grant NSC 992115-M-110-007-MY3 and Grant NSC 99-2221-E-037-007-MY3, respectively.

\section{Author details}

${ }^{1}$ Department of Mathematics, Yasouj University, Yasouj 75918, Iran ${ }^{2}$ Department of Applied Mathematics, National Sun Yat-Sen University, Kaohsiung 804, Taiwan ${ }^{3}$ Center for General Education, Kaohsiung Medical University, Kaohsiung 807, Taiwan

\section{Authors' contributions}

All authors contribute equally and significantly in writing this paper. All authors read and approved the final manuscript.

\section{Competing interests}

The authors declare that they have no competing interests.

Received: 10 June 2011 Accepted: 23 November 2011 Published: 23 November 2011

\section{References}

1. Nadezhkina, N, Takahashi, W: Strong convergence theorem by a hybrid method for nonexpansive mappings and Lipschitz continuous monotone mappings. SIAM J Optim. 16, 1230-1241 (2006). doi:10.1137/050624315

2. Rockafellar, RT: Monotone operators and the proximal point algorithm. SIAM J Control Optim. 14, 877-898 (1976). doi:10.1137/0314056

3. Rockafellar, RT: On the maximality of sums of nonlinear monotone operators. Trans Am Math Soc. 149, 75-88 (1970). doi:10.1090/S0002-9947-1970-0282272-5

4. Goebel, K, Kirk, WA: A fixed point theorem for asymptotically nonexpansive mappings. Proc Am Math Soc. 35, 171-174 (1972). doi:10.1090/S0002-9939-1972-0298500-3 
5. Sahu, DR, Xu, H-K, Yao, J-C: Asymptotically strict pseudocontractive mappings in the intermediate sense. Nonlinear Anal. 70, 3502-3511 (2009). doi:10.1016/j.na.2008.07.007

6. Kinderlehrer, D, Stampacchia, G: An Introduction to Variational Inequalities and their Applications. Academic Press, New York (1980)

7. Korpelevich, GM: The extragradient method for finding saddle points and other problems. Matecon. 12, 747-756 (1976)

8. Ceng, LC, Sahu, DR, Yao, JC: Implicit iterative algorithms for asymptotically nonex-pansive mappings in the intermediate sense and Lipschitz continuous monotone mappings. J Comput Appl Math. 233, 2902-2915 (2010). doi:10.1016/j. cam.2009.11.035

9. Ceng, L-C, Hadjisavvas, N, Wong, N-C: Strong convergence theorem by a hybrid extragradient-like approximation method for variational inequalities and fixed point problems. J Global Optim. 46, 635-646 (2010). doi:10.1007/s10898009-9454-7

10. Goebel, K, Kirk, WA: Topics on Metric Fixed-Point Theory. Cambridge University Press, Cambridge (1990)

doi:10.1186/1029-242X-2011-119

Cite this article as: Naraghirad et al:: Strong convergence theorems by a hybrid extragradient-like approximation method for asymptotically nonexpansive mappings in the intermediate sense in Hilbert spaces. Journal of Inequalities and Applications 2011 2011:119.

\section{Submit your manuscript to a SpringerOpen ${ }^{\circ}$} journal and benefit from:

- Convenient online submission

- Rigorous peer review

- Immediate publication on acceptance

- Open access: articles freely available online

- High visibility within the field

- Retaining the copyright to your article

Submit your next manuscript at $\gg$ springeropen.com 